\title{
STOCHASTIC VORTEX BLOBS METHOD TO STUDY AXISYMMETRIC FLOWS OF INCOMPRESSIBLE VISCOUS FLUID
}

\author{
M. R. Hedar and R. S. Kamel \\ Mathematical Department, Faculty of Science, Minia University, El-Minia, EGYPT \\ mrhedar@yahoo.com \\ Rehab.shaher@yahoo.com
}

\begin{abstract}
This paper presents the mathematical and computational formulations of the stochastic Vortex Blobs Method (VBM). It is show that how the method can be used to cover axisymmetric flows of incompressible viscous fluid. Also, the initialboundary problem is solved by using the Lagrangian vortex method. This method seems to be an extension of the well known two dimensional vortex blob method. When applying and extending this method two steps are required. First, we have to design an axisymmetric vorticity carrier by using the standard functions as complete elliptic integrals and Legendre polynomials. Second, it is necessary to formulate the appropriate Neumann problem and boundary integral equation to find the potential velocity fields. Both steps are used to describe and compute the total velocity field and formulate the lto stochastic equations which describing the motion of vorticity carriers.
\end{abstract}

\section{Mathematics Subject Classification: 74F10, 76, 76A02 and 80}

Keywords: Vortex blobs method; axisymmetric flow, Fokker-Plank-Kolmogorow equation; Ito stochastic differential equations; Elliptic integrals; Legendre polynomials; Hankel transforms.

\section{INTRODUCTION}

The study of the flow field in case of an axisymmetric incompressible viscous fluid by using stochastic approach will be presented. It seems to be the same idea and an extension of the Hedar and Styczek [6] paper concerning the random vortex method approach to axisymmetric jet in a large tank. In that paper the vortex blobs method has been applied to description of an axisymmetric viscous flow. Also, they presented the stochastic interpretation of the vorticity equation which leads to the Ito differential equations determining the movement of large numbers of the blobs. The main idea of that is to approximate the vorticity by a large set of small vortex creatures called vortex blobs being transported in velocity field and performing random walks according to Wiener process. The velocity field evolves in time due to change of vorticity distribution. In order to satisfy the boundary conditions, new vortex blobs are continuously created on the boundary. Of course, the velocity field contains also a proper potential component. This component allows formulating boundary integral equation for the vorticity created at the moment. Thus, the Neumann boundary problem should be solved. Suitable considerations can be found in the papers $[7,10]$. This is showed how to reduce the problem to the boundary integral equation with the vorticity distribution on the boundary as an unknown function.

Generally, the vortex method is a type of numerical methods for approximating solution of Helmholtz's equation. This method can be regarded as a discretization of this equation in a special form. The vortex method brings some advantages as the result of elimination of pressure and is believed to belong to a new class of powerful numerical techniques for simulation of fluid motion. Chorin [3] was first to propose the vortex blobs approach. Since that time the formulation of random vortex method became a standard. Styczek [10] proposed a new approach to the boundary conditions. The results can be found in the papers published latter $[11,12,13,14]$. This method is useful especially when the vorticity is created in small regions of the domain.

Theoretically the vortex motion forms the continuous family of Ito's stochastic processes governed by Ito's stochastic differential equations. Of course, this continues family must be cut off to finite one. In an axisymmetric viscous flow, this method requires to study the mathematical and computational formulations of the problem. This enables us to study the construction of axisymmetric vorticity carrier and find the solution of potential velocity field. Finally, the formulations of Ito stochastic differential equations describing the motion of vorticity carriers are considered. The complete details and final results of this problem will be formulated and discuss in next paper.

\section{FORMULATION OF THE PROBLEM}

For an incompressible viscous fluid, any flow must be satisfying the governing equations. They are "conservation equation of mass" and "momentum equation", which they written as

$\operatorname{div} \vec{V}=0$,

$\frac{d \vec{V}}{d t}=\underline{\mathbf{F}}-\frac{1}{\rho} \operatorname{grad} p+v \Delta \vec{V}$ 
where $\vec{V}, p, \rho$ stand for velocity vector, pressure and density $(\rho=1)$. The kinematic viscosity coefficient is denoted by $U$ and $\underline{\mathbf{F}}$ represents the external body force field.

In the case of an axisymmetric incompressible viscous fluid, we assume that the external body force is negligible $(\underline{\mathbf{F}}=0)$ and consider the task of determining the velocity field. We have velocity vector with radial and axial components $\vec{V}=\left\{V_{r}(t, r, z), V_{z}(t, r, z)\right\}$. In this case the above system of equations can be written as

$$
\begin{aligned}
& \frac{\partial V_{r}}{\partial r}+\frac{\partial V_{z}}{\partial z}+\frac{V_{r}}{r}=0, \\
& \frac{\partial V_{r}}{\partial t}+V_{r} \frac{\partial V_{r}}{\partial r}+V_{z} \frac{\partial V_{r}}{\partial z}=-\frac{\partial p}{\partial r}+v\left[\frac{\partial^{2} V_{r}}{\partial r^{2}}+\frac{\partial^{2} V_{r}}{\partial z^{2}}+\frac{1}{r} \frac{\partial V_{r}}{\partial r}-\frac{V_{r}}{r^{2}}\right], \\
& \frac{\partial V_{z}}{\partial t}+V_{r} \frac{\partial V_{z}}{\partial r}+V_{z} \frac{\partial V_{z}}{\partial z}=-\frac{\partial p}{\partial z}+v\left[\frac{\partial^{2} V_{z}}{\partial r^{2}}+\frac{\partial^{2} V_{z}}{\partial z^{2}}+\frac{1}{r} \frac{\partial V_{z}}{\partial r}\right]
\end{aligned}
$$

The equations (2.1) are known continuity and Navier - Stokes equations, which they are valid for $(t>0, r>0, z \geq 0)$. The initial-boundary data can be formulated as

- There is no motion at the initial instant $(t=0)$ and by setting the constraints $(z=0)$ at the boundary we can write the set of equations of initial-boundary data in the form

$$
\begin{aligned}
& \vec{V}(0, r, z)=0 \\
& V_{r}(t, r, 0)=0 \\
& V_{z}(t, r, 0)=f(r)
\end{aligned}
$$

- We can also impose the symmetry condition for the velocity $V_{r}(t, 0, z)=0$ and the requirement for tangential stress at $r=0$ is $\left(\left.\frac{\partial V_{z}}{\partial r}\right|_{r=0}=0\right)$.

It may be noticed that there is no boundary condition imposed on pressure field. It can be evaluated when the gradient of pressure field is known in the entire flow domain. It means that velocity field and pressure field can be determined independently. Having calculated velocity and vorticity fields it is possible to find $(\operatorname{grad} p)$ and then the function $p$ itself.

According to the theory of vortex method we introduce the vorticity field $(\vec{\omega}=\operatorname{rot} \vec{V})$. For axisymmetric flow $\left(\frac{\partial}{\partial \theta}=0\right)$, there is only one non-zero component of vorticity $\omega$. This component is directed in $\theta$ direction which it is has the value

$\omega=\left(0, \omega_{\theta}, 0\right)=\left(\frac{\partial V_{r}}{\partial z}-\frac{\partial V_{z}}{\partial r}\right)$

It is well known that the vorticity satisfies the Helmholtz equation

$\frac{\partial \omega}{\partial t}+\frac{\partial}{\partial r}\left[\left(V_{r}-\frac{v}{r}\right) \omega\right]+\frac{\partial}{\partial z}\left[V_{z} \omega\right]=v\left[\frac{\partial^{2} \omega}{\partial r^{2}}+\frac{\partial^{2} \omega}{\partial z^{2}}\right]$

After substitution $\left[r=y, V_{y}=\left(V_{r}-\frac{v}{r}\right)\right]$ in the equation (2.4), we obtain the well known Planck-Fokker-Kolmogorow equation [5]

$\frac{\partial \omega}{\partial t}+\frac{\partial}{\partial y}\left(V_{y} \omega\right)+\frac{\partial}{\partial z}\left(V_{z} \omega\right)=v \nabla^{2} \omega$

Function $\omega$ can be represented in the integral form

$\omega(t, y, z)=\int \mathbf{p}\left(t, y, z \mid 0, y_{0}, z_{0}\right) \omega_{0}\left(y_{0}, z_{0}\right) d y_{0} d z_{0}$

where $\omega_{0}\left(y_{0}, z_{0}\right)$ describes initial vorticity field and $\mathbf{p}$ is a transition probability density function of the family of certain stochastic process.

The diffusion process determines the infinitesimal element $\Delta \vec{r}$

$\Delta \vec{r}=\vec{V}(t, \vec{r}) \Delta t+\mathbf{A}(t, \vec{r}) \Delta \vec{W}(\Delta t)$

in which the vector $\vec{r}$ consists of components $(y, z), \mathbf{A}$ is the symmetric matrix $\mathbf{A}: \mathbf{A}^{i i}=2 v$ and $\mathbf{A}^{i k}=0$ for $i \neq k$, the vector $\Delta \vec{W}$ is the increase of Wiener process [10]. These processes are described by lto equations 
$d y=V_{y} d t+\sqrt{2 v} d W_{y}$

$d z=V_{z} d t+\sqrt{2 v} d W_{z}$

with the initial conditions $\left(\left.y\right|_{t=0}=y_{0},\left.\quad z\right|_{t=0}=z_{0}\right)$.

The family of process can be interpreted as the motion in $(y-z)$ plane. At the initial instant of time the process starts at the point $\left(y_{0}, z_{0}\right)$ and reaches the point $(y, z)$ during the time $t$. Next, we write

$$
\mathbf{P}\left(t, x, y \in A \mid x_{l_{0}}=x_{0}, y_{\left.\right|_{0}}=y_{0}\right)=\int_{A} \mathbf{p}\left(t, x, y \mid 0, x_{0}, y_{0}\right) d x d y
$$

The transition probability density function $\mathbf{p}$ of the family of certain stochastic process satisfies Planck-FokkerKolmogorow equation [5]

$\frac{\partial \mathbf{p}}{\partial t}+\frac{\partial}{\partial y}\left(V_{y} \mathbf{p}\right)+\frac{\partial}{\partial z}\left(V_{z} \mathbf{p}\right)=v \nabla^{2} \mathbf{p}$

We can formulate equation (2.6) as a result of ordinary convolution of $\mathbf{p}$ and the initial data.

The whole plane into elementary subregions $\Omega_{m}$ can be divided and we can approximate the transition probability density function $\mathbf{p}$ in the way

$\mathbf{p}\left(t, y, z \mid 0, y_{0}, z_{0}\right)=\sum_{(m)} \mathbf{p}_{m}(t, y, z) \chi_{m}\left(y_{0}, z_{0}\right)$

where $\mathbf{p}_{m}$ denotes $\mathbf{p}_{m}(t, y, z)=\mathbf{p}\left(t, y, z \mid 0, y_{0 m}, z_{0 m}\right),\left(y_{0 m}, z_{0 m}\right) \in \Omega_{m}$ and $\chi_{m}$ is the characteristic function of $\Omega_{m}$. Thus the vorticity written as

$$
\omega(t, y, z)=\sum_{(m)} \mathbf{p}_{m}(t, y, z) \int_{\Omega_{m}} \omega_{0}\left(y_{0}, z_{0}\right) d y_{0} d z_{0}
$$

Associating the movement of a point $\left(y_{o m}, z_{o m}\right)$ with the movement of a small region $\Omega_{m}$ we get motion of elementary vorticity carrier. This carrier transports a small charge of vorticity

$\Gamma_{m}=\int_{\Omega_{m}} \omega_{0}\left(y_{0}, z_{0}\right) d y_{0} d z_{0}$

The charge of vorticity $\Gamma_{m}$ and $\mathbf{p}_{m}(t, y, z)$ define vorticity

$\omega(t, y, z)=\sum_{(m)} \Gamma_{m} \mathbf{p}_{m}(t, y, z)$

This interpretation brings us the vortex blobs understood as small, moving carriers of the vorticity.

We consider the outline of the area of motion filled with vorticity carriers. They move and are continuously created on the boundary. Vorticity carriers created previously are carrying with the known charge of vorticity. The position of each carrier is known and results from the solution of the set equations (2.8) which becomes

$d y_{m}=\left.V_{y}\right|_{y_{m}, z_{m}} d t+\sqrt{2 v} d W_{y_{m}}$

$d z_{m}=\left.V_{z}\right|_{y_{m}, z_{m}} d t+\sqrt{2 v} d W_{z_{m}}$

The initial conditions complete the equation (2.12). They express the position of the carriers at the moment $t_{\text {om }}$ when they were created $\left(\left.y_{m}\right|_{t_{0 m}}=y_{0 m},\left.z_{m}\right|_{t_{o m}}=z_{0 m}\right)$. This point $\left(y_{0 m}, z_{0 m}\right)$ defines the position where carrier is created. There are also carriers created at the given moment. These are indicated as " $\mathrm{n}$ " (new) class and the others carriers are called "o" (old) were created before. In order to determine velocity field we have to take into account all carriers being in the domain. Furthermore, velocity field must contain a potential term. Let us write the velocity field in the following form

$\vec{V}=\vec{V}_{p}+\vec{V}_{n}+\vec{V}_{o}+\vec{V}_{s}+\vec{V}_{A}$

where $\vec{V}_{p}$ and $\vec{V}_{A}$ are potential velocity fields, $\vec{V}_{n}$ velocity field induced by the carriers marked with "n" (new blobs), $\vec{V}_{o}$ velocity field induced by the carriers marked with "o" (old blobs) and $\vec{V}_{s}$ is the self induced velocity.

We define the velocity fields mentioned above. First, we define the potential term: 
$\nabla^{2} \Phi_{p}=0, \quad V_{p}=\nabla \Phi_{p},\left.\quad \frac{\partial \Phi_{p}}{\partial n}\right|_{z=0}=f(r)$

Equations (2.14) defining Neumann problem where the function $f(r)$ has been defined with the boundary condition (2.2). The solution of this problem will be discussed in section four.

The velocity field $\vec{V}_{o}$ has the form

$\vec{V}_{o}=\sum_{(m)} \Gamma_{m} \vec{V}\left(t, r, z, r_{m}, z_{m}\right)$

The velocity field $\vec{V}\left(t, r, z, r_{m}, z_{m}\right)$ is induced by a carrier with the unit charge of vorticity. This carrier is located at the surroundings of the point with coordinates $\left(r_{m}, z_{m}\right)$. The charge $\Gamma_{m}$ is constant and known. The velocity field $\vec{V}_{n}$ has the form

$\vec{V}_{n}=\sum_{(k)} \Gamma_{k} \overrightarrow{\mathrm{V}}\left(r, z, r_{k}, z_{k}\right)$

The charge $\Gamma_{k}$ is unknown and $\overrightarrow{\mathrm{V}}\left(r, z, r_{k}, z_{k}\right)$ denotes the velocity induced by the carrier boring at the moment which is located at known point. Here velocity field $\overrightarrow{\mathrm{V}}$ does not depend on time, because time increment is zero when appropriate carrier is created at the moment.

The last term $\vec{V}_{A}$ of equation (2.13) results from equations (2.14) which define the additional potential in the form

$$
\nabla^{2} \Phi_{A}=0 \quad, \quad \vec{V}_{A}=\nabla \Phi_{A} \quad,\left.\quad \frac{\partial \Phi_{A}}{\partial z}\right|_{z=0}=V_{A z}(r)
$$

It is the velocity field $V_{A z}(r)$ which causes that normal component of the total velocity field to be equal to zero $(\vec{V} \cdot \vec{n}=0)$ on the line $(z=\mathbf{O})$.

If we consider that self induced velocity is not found and by using the boundary conditions (2.2), the terms of velocity field components can be expressed in the form

$V_{p r}(r, 0)+V_{n r}(r, 0)+V_{o r}(t, r, 0)+V_{A r}(r, 0)=0$,

$V_{n z}(r, 0)+V_{o z}(t, r, 0)+V_{A z}(r, 0)=0$

Function $f(r)$ was eliminated due to the features of the velocity field $\vec{V}_{p}$. Both the components $V_{o r}$ and $V_{o z}$ are known. Also the component $V_{p r}$ is completely defined by means of the potential $\Phi_{p}$ which is a solution of the problem (2.14). The components $V_{A z}$ and $V_{A r}$ are determined as the derivatives of the potential $\Phi_{A}$ which is unknown. Taking into account that the components $V_{o r}$ and $V_{o z}$ are known apart from $\Phi_{A}$ we have to calculate the vorticity distribution induced by the "n" class of vortices. Of course, the components $V_{n r}$ and $V_{n z}$ are unknown. But they are defined by boundary value of vorticity. There are two equations with two unknown functions. One of them is the boundary value of vorticity and the second is the component $V_{A z}$. It is possible to solve this problem. If these have been done, then the total velocity may be found. Then, all blobs move according to Ito's equations (2.8).

The idea of the vortex method consists in the calculation of above mentioned quantities. After determination of these variables we include the new class "n" of carriers into the old class "o". Having calculated the velocity field we move the carriers according to Ito equations (2.8). In each instant of time there are new " $n$ " classes of carriers to be generated. In the first instant of time there is no movement of the fluid and of course vortices of class "o" do not exist. This procedure is repeated again and again and it enables to evaluate time history of vorticity and velocity fields. As it seems to us, the present study will be complete to give the details, discussions and final results of this procedure in next paper.

\section{AXISYMMETRIC VORTICTY CARRIER}

For an axisymmetric incompressible viscous fluid flows, there is a relationship which defines the vorticity field $\omega$ given in equation (2.3). The velocity field must be satisfying the continuity equation

$\frac{\partial\left(r V_{r}\right)}{\partial r}+\frac{\partial\left(r V_{z}\right)}{\partial z}=0$ 
This formula enables to find velocity field induced by vorticity. To obtain one equation with one unknown we introduce new unknown Stokes stream function $\psi$. We have

$V_{r}=-\frac{1}{r} \frac{\partial \psi}{\partial z} \quad, \quad V_{z}=\frac{1}{r} \frac{\partial \psi}{\partial r}$

It is easy to notice that velocity components (3.2) reduce continuity equation to identity. Definition of vorticity leads to elliptic Poisson equation

$\frac{\partial^{2} \psi}{\partial r^{2}}-\frac{1}{r} \frac{\partial \psi}{\partial r}+\frac{\partial^{2} \psi}{\partial z^{2}}=-r \omega$

Since the hypothesis of an axisymmetric flow is used [9], the vorticity field is discretized using vortex elements in the form of vortex blobs. For an infinite domain, there is an analytical expression for the solution of equation (3.3) when the vorticity field is discretized with vortex blobs. The stream function induced by a vortex ring with circulation $\Gamma$ and radius $\sigma$ on a point located at an axial distance $z$ and a radial distance $r$ from the center of the ring is given by the expression

$\psi=\left(\frac{-r \Gamma \sigma}{4 \pi}\right) \int_{0}^{2 \pi} \frac{\cos \alpha d \alpha}{\sqrt{r^{2}+\sigma^{2}-2 r \sigma \cos \alpha+(z-\eta)^{2}}}$

where the definition of circulation is $\left(\Gamma=\iint \omega d \sigma d \eta\right)$ and by setting

$\int_{0}^{2 \pi} \frac{\cos \alpha d \alpha}{\sqrt{r^{2}+\sigma^{2}-2 r \sigma \cos \alpha+(z-\eta)^{2}}}=\frac{2}{\sqrt{r \sigma}}\left[\left(\frac{2}{k}-k\right) K(k)-\frac{2}{k} E(k)\right], \quad k=\left[\frac{4 r \sigma}{(z-\eta)^{2}+(\sigma+r)^{2}}\right]^{\frac{1}{2}}$

Equation (3.4) can be written in the form

$\psi=\frac{-\Gamma \sqrt{\sigma r}}{2 \pi}\left[\left(\frac{2}{k}-k\right) K(k)-\frac{2}{k} E(k)\right]$

While $K(k)$ and $E(k)$ are the complete elliptic integrals from the first and second kinds belong to standard function are given by the following relations respectively

$K(k)=\int_{0}^{\frac{\pi}{2}} \frac{d \phi}{\sqrt{1-k^{2} \sin ^{2} \phi}} \equiv \frac{\pi}{2} \sum_{n=0}^{\infty}\left[p_{2 n}(0)\right]^{2} k^{2 n}$

$E(k)=\int_{0}^{\frac{\pi}{2}} \sqrt{1-k^{2} \sin ^{2} \phi} d \phi \equiv \frac{\pi}{2} \sum_{n=0}^{\infty}\left[p_{2 n}(0)\right]^{2}\left(\frac{k^{2 n}}{1-2 n}\right)$

where $p_{2 n}$ is Legendre polynomials.

The components of velocity induced by the vortex blob are obtained by using the equations $\{(3.2),(3.5)\}$ as the following

$V_{r}(r, z \mid \xi, \eta, \sigma)=\left(\frac{\Gamma k(z-\eta)}{4 \pi r \sqrt{\sigma r}}\right)\left[-K(k)+\left(\frac{r^{2}+\sigma^{2}+(z-\eta)^{2}}{(z-\eta)^{2}+(\sigma-r)^{2}}\right) E(k)\right]$,

$V_{z}(r, z \mid \xi, \eta, \sigma)=\left(\frac{\Gamma k}{4 \pi \sqrt{\sigma r}}\right)\left[K(k)+\left(\frac{\sigma^{2}-r^{2}-(z-\eta)^{2}}{(z-\eta)^{2}+(\sigma-r)^{2}}\right) E(k)\right]$

The system of equations $\{(3.5)-(3.7)\}$ can be calculated numerically with high level accuracy to obtain the stream function and induced velocity field for one vortex blob.

The linearity of all equations makes it possible to invoke the principal of superposition to account for the presence of multiple point vortices in the flow. Tagging each point vortex by subscript $i$ and $j$, we can immediately repeat the expression of the stream function in equation (3.5) and the components of velocity field in equations (3.7) on the forms

$\psi=\frac{-\Gamma \sqrt{\sigma_{i} r}}{2 \pi}\left[\left(\frac{2}{k}-k\right) K(k)-\frac{2}{k} E(k)\right], k=\sqrt{\frac{4 r \sigma_{i}}{\left(z-\eta_{j}\right)^{2}+\left(\sigma_{i}+r\right)^{2}}}$,

$V_{r}\left(r, z \mid \xi, \eta_{j}, \sigma_{i}\right)=\frac{\Gamma k\left(z-\eta_{j}\right)}{4 \pi r \sqrt{\sigma_{i} r}}\left[-K(k)+\left(\frac{r^{2}+\sigma_{i}^{2}+\left(z-\eta_{j}\right)^{2}}{\left(z-\eta_{j}\right)^{2}+\left(\sigma_{i}-r\right)^{2}}\right) E(k)\right]$,

$V_{z}\left(r, z \mid \xi, \eta_{j}, \sigma_{i}\right)=\frac{\Gamma k}{4 \pi \sqrt{\sigma_{i} r}}\left[K(k)+\left(\frac{\sigma_{i}^{2}-r^{2}-\left(z-\eta_{j}\right)^{2}}{\left(z-\eta_{j}\right)^{2}+\left(\sigma_{i}-r\right)^{2}}\right) E(k)\right]$

The system of equations $\{(3.8)-(3.9)\}$ can be calculated numerically with high level accuracy to obtain the stream function and induced velocity field for multi-vortices.

\section{POTENTIAL VELOCITY FIELD}


Generally, to find the potential velocity field we solve the Laplace equation (2.14) which becomes in an axisymmetric case as the following

$$
\begin{aligned}
& \frac{\partial^{2} \Phi}{\partial r^{2}}+\frac{1}{r} \frac{\partial \Phi}{\partial r}+\frac{\partial^{2} \Phi}{\partial z^{2}}=0, \quad r, z>0 \\
& \left.\frac{\partial \Phi}{\partial z}\right|_{z=0}=f(r) \quad, \quad V_{r}=\frac{\partial \Phi}{\partial r} \quad, \quad V_{z}=\frac{\partial \Phi}{\partial z}
\end{aligned}
$$

The simpler way to obtain closed formula expressing potential field $\Phi$ is the Hankel transform method [1]. Thus, multiplying Laplace's equation by $\left[r_{0}(s r)\right]$ and integrating with respect to $r$ over the interval $[0, \infty)$ we get

$\frac{d^{2} \hat{\Phi}}{d z^{2}}-s^{2} \hat{\Phi}=0$

Here $\hat{\Phi}$ denotes Hankel transform and further $J_{k}$ stands for ordinary Bessel function. Then the integral solution of equation (4.2) is

$\hat{\Phi}(s, z)=\int_{0}^{\infty} \Phi(r, z) r J_{0}(s r) d r$

Inverse transform is calculated as follows

$\Phi(r, z)=\int_{0}^{\infty} \hat{\Phi}(s, z) s J_{0}(s r) d s$

The particular solution of the equation (4.2) which vanishes for $z \rightarrow \infty, s>0$ has the form $\left(\hat{\Phi}(s, z)=C e^{-s z}\right)$ and contains the transform of the function $f(r)$ as

$\hat{f}(s)=\int_{0}^{\infty} f(r) r J_{0}(s r) d r$

For particular solution $f(r)$ can be defined by the equation

$f(r, a)= \begin{cases}1 & r<a \\ 0 & r>a\end{cases}$

In this case we obtain

$\hat{f}(s)=\int_{0}^{a} r J_{0}(r s) d r=\frac{a}{s} J_{1}(a s)$

Having determined boundary condition we have $\left(C=-\frac{a}{s^{2}} J_{1}(a s)\right)$, thus

$\Phi(r, z)=-a \int_{0}^{\infty} J_{0}(r s) J_{1}(a s) e^{-s z} \frac{d s}{s}$

Differentiating equation (4.7) enables to write

$V_{r}=a \int_{0}^{\infty} J_{1}(r s) J_{1}(a s) e^{-s z} d s$

$V_{z}=a \int_{0}^{\infty} J_{0}(r s) J_{1}(a s) e^{-s z} d s$

These components define the potential velocity field.

We should notice that for $(z>0)$ the integrals are convergent. This fact results from the limitation of the absolute values of Bessel function and exponentially vanishing damping factor $\left(e^{-s z}\right)$. After calculating the transform $\hat{f}(s)$ we are able to solve the Neumann problem for arbitrary boundary condition $\left.\frac{\partial \Phi}{\partial z}\right|_{z=0}=f(r)$. For plane $(z=0)$ the function $\Phi$ has the form

$\Phi(r, 0)=-a \int_{0}^{\infty} J_{0}(r s) J_{1}(a s) \frac{d s}{s}$

The convergence of this integral may be slow. Then it is necessary to apply accurate calculus.

\section{THE COMPUTATION METHOD}


To compute the complete velocity field (2.13), we use the boundary integral equation and by returning to the Neumann problem (4.1), where the function $f(r)$ is specified completely by the potential $\Phi$ and $\left.\frac{\partial \Phi}{\partial n}\right|_{z=0}=\left.\frac{\partial \Phi}{\partial z}\right|_{z=0}$. For regular boundary such as the plane $(z=0)$ there exist the following formulas

$$
\begin{aligned}
& \left.V_{z}\right|_{z=0}=\left.(\vec{n} \cdot \nabla \Phi)\right|_{z=0}=\left.\frac{\partial \Phi}{\partial z}\right|_{z=0} \\
& \left.V_{r}\right|_{z=0}=\left.(\vec{t} \cdot \nabla \Phi)\right|_{z=0}=\left.\frac{\partial \Phi}{\partial r}\right|_{z=0}
\end{aligned}
$$

Neumann problem (4.1) is linear; therefore a linear operator $\boldsymbol{L}$ exists and satisfies equation

$$
\left.V_{r}\right|_{z=0}=\left.\frac{\partial \Phi}{\partial r}\right|_{r=0}=L\left(\left.\frac{\partial \Phi}{\partial z}\right|_{z=0}\right)=L(F)
$$

Operator $L$ has an integral form and is singular [10], thus

$$
\left.\int_{r_{i}}^{r_{j}} V_{r}\right|_{z=0} d r=\int_{r_{i}}^{r_{j}} L(F) d r=\left.\int_{r_{i}}^{r_{j}} \frac{\partial \Phi}{\partial r}\right|_{z=0} d r
$$

By using Schauder theorem [2] we express the equation (5.3) as the increment of potential

$\left.\int_{r_{i}}^{r_{j}} \frac{\partial \Phi}{\partial r}\right|_{z=0} d r=\Phi\left(r_{j}, 0\right)-\Phi\left(r_{i}, 0\right)$

In order to transform equations (2.16) we apply the relationships given here. By expressing $\left.V_{A z}\right|_{z=0}=\left.\frac{\partial \Phi_{A}}{\partial z}\right|_{z=0}$ in the second equation and applying the operator $L$ we can obtain $\left.V_{A r}\right|_{z=0}$. Then substituting $V_{A r}$ in the first equation one gets the following integral equation

$V_{p r}(r, 0)+V_{n r}(r, 0)+V_{o r}(t, r, 0)-L\left(V_{n z}(r, 0)\right)-L\left(V_{o z}(t, r, 0)\right)=0$

Thus, there is only one unknown function in equation (5.5). It is boundary density of vorticity which determines boundary values of the field $\vec{V}_{n}$. The above equation (5.5) written as

$V_{n r}(r, 0)-L\left[V_{n z}(r, 0)\right]=L\left[V_{o z}(t, r, 0)\right]-V_{o r}(t, r, 0)-V_{p r}(r, 0)$

Right hand side of equation (5.6) is known and left hand side of it contains the components $V_{n r}$ and $V_{n z}$ which will be calculated in next section.

The solution of equation (5.6) enables to calculate the vorticity charges of new created vortices and at the same time the velocity satisfies boundary conditions. This solution is not easy in numerical calculation due to singular character of the operator $\boldsymbol{L}$ [10], however there is a possibility to regularize equation (5.6). Namely, by integrating with respect to $r$ over the interval $\left(r_{i}, r_{i+1}\right)$ and using the properties of operator $L$, we obtain

$\int_{r_{i}}^{r_{i+1}} V_{n r}(\zeta, 0) d \zeta-\delta_{i}^{i+1} \Phi_{n}=\delta_{i}^{i+1} \Phi_{o}-\int_{r_{i}}^{r_{i+1}} V_{o r}(t, \zeta, 0) d \zeta-\delta_{i}^{i+1} \Phi_{p}$

The symbol $\left(\delta_{i}^{i+1} \Phi\right)$ is used to indicate potential increment over the section $\left(r_{i}, r_{i+1}\right)$ of the plane $(z=0)$. We introduce the components $V_{n r}$ and $V_{n z}$ according to the formula (2.16).

Moreover, we denote

$V_{z}\left(t, r, 0, r_{k}, z_{k}\right)=f_{k}(r)$

and evaluate the potential $\Phi_{n k}$ from the potential term

$\nabla^{2} \Phi_{n k}=0 \quad,\left.\quad \frac{\partial \Phi_{n k}}{\partial z}\right|_{z=0}=f_{k}(r)$

Then we are able to write 


$$
\begin{aligned}
& \sum_{(k)} \Gamma_{k}\left\{\int_{r_{i}}^{r_{i+1}} V_{n r}\left(\zeta, 0, r_{k}, z_{k}\right)-\delta_{i}^{i+1} \Phi_{n k}\right\}=G_{i}, \\
& G_{i}=\delta_{i}^{i+1} \Phi_{o}-\delta_{i}^{i+1} \Phi_{p}-\int_{r_{i}}^{r_{i+1}} V_{o r}(t, \zeta, 0) d \zeta
\end{aligned}
$$

Equation (5.10) it is represents the set of linear algebraic equations which enables to calculate the charge of vorticity of new vorticity carriers.

Thus, all components of the total velocity field $\vec{V}(t, r, z)$ are known except for the circulation of velocity field for new blobs $\vec{V}_{n}$. The circulations of new blobs which are created on the boundary are $\left\{\Gamma_{1}, \Gamma_{2}\right.$ $\left.\Gamma_{k}\right\}$. The circulations obtained from the boundary integral equation for the boundary vorticity distribution by using the set of equations (5.10). The components of velocity field $\vec{V}_{n}$ can be defined as

$$
\begin{aligned}
& V_{n r}(r)=\sum_{(k)} \Gamma_{k} T_{k}(r) \\
& V_{n z}(r)=\sum_{(k)} \Gamma_{k} N_{k}(r)
\end{aligned}
$$

Both functions $T_{k}$ and $N_{k}$ are described by geometrical properties of blobs. These functions take the same formulas of $V_{O r}$ and $V_{O z}$ at the boundary.

To determine the circulations $\Gamma_{k}$, the system of equations (5.10) can be rewritten as:

$\sum_{(k)} \Gamma_{k} B_{i k}=G_{i}$

The unknown vector $\Gamma_{k}$ in equation (5.12) describes the scale of vorticity inside the boundary blobs, $B_{i k}$ define the elements of matrix and $G_{i}$ define the free terms [4]. Thus, the values of scale $\Gamma_{k}$ written as

$\Gamma_{k}=\sum_{(k)}\left[B_{i k}\right]^{-1} G_{i}$

This system is introduced due to the vorticity creation on the boundary. By solving this system we obtain the circulations for new blobs.

To solve the system (5.13) we assume that the geometry of the flow does not change in time, so that the matrix $B_{i k}$ can be calculated and factorized in advance. It means that the matrix can be calculated only once and the boundary vortex array must be defined at the time-stepping calculations. Then the velocity induced by the vorticity field is known. So, it is possible to determine the velocity induced by carriers of the class " $n$ " and class "o". There is no difference in the way of calculating this induction.

We simply sum up the contributions from the complete set of carriers

$\vec{V}_{n}+\vec{V}_{o}=\vec{V}_{w}=\sum_{(k)} \Gamma_{k} \vec{V}\left(r, z, r_{k}, z_{k}\right)$

Finally, we find $\Phi_{A}$, and as a result the additional velocity field $\vec{V}_{A}$ by evaluating the boundary value of $V_{A z}(t, r, 0)$. To obtain this field we use equation (5.5) and solving Neumann problem (2.15). Because the velocity field $\vec{V}_{p}$ is already known it is enough to add the remaining components and obtain the complete velocity field $\vec{V}(t, r, z)$ which is responsible for the motion of carriers.

\section{RESULTS OF THE COMPUTATION}

The tangent and normal components $V_{r}(t, r, z)$ and $V_{z}(t, r, z)$ of the complete velocity field are obtained from equation (2.13). By using equation (5.14) the components of this field can be rewritten as

$$
\begin{aligned}
& V_{r}(t, r, z)=V_{p r}+V_{w r}+V_{A r} \\
& V_{z}(t, r, z)=V_{p z}+V_{w z}+V_{A z}
\end{aligned}
$$

Then all terms of total velocity field are given. 
Finally, after calculating the total velocity field, we determine the positions of the blobs which they are borne at each time steps and they are moves with flow doing random walks. These blobs are consists in numerical integration of lto equations (2.8). The Ito's equations can be found as the numerical process by using the Euler algorithm [8]. Thus, the system of Ito's equations can be written numerically as the following

$$
\begin{aligned}
& r_{i+1}(t+\Delta t)=r_{i}(t)+\left[V_{r}\left(t, r_{i}, z_{i}\right)-\frac{v}{r_{i}(t)}\right] \Delta t+\sqrt{2 v \Delta t} N_{1} \\
& z_{i+1}(t+\Delta t)=z_{i}(t)+\left[V_{z}\left(t, r_{i}, z_{i}\right)\right] \Delta t+\sqrt{2 v \Delta t} N_{2}
\end{aligned}
$$

where $\left(N_{1}, N_{2}\right)$ denote the random numbers with Gaussian distribution $N(0,1)$.

The system of equations (6.2) reduces the Wiener process to the standard form. Therefore, the solution of the vorticity transport equation is performed by imposing stochastic displacements of the vortex blobs in two orthogonal directions. Each displacement is generated randomly from two sets of independent Gaussian random numbers, with each set having a zero mean and a standard deviation $(\sqrt{2 v \Delta t})$. Performing random walks at each time step and adding the displacements to obtain the total displacement at time $t$ is possible. The centroids of the blobs are located over centers of corresponding segments of r-axis. The blobs change locations due to the numerical system of Ito's equations (6.2). Some of blobs are canceled. The test for cancellation is $\left(r_{i}<0, z_{i}<0\right)$, where $\left(r_{i}, z_{i}\right)$ denote the location of blob centroid. Thus, the numerical calculations and the results can be computed.

As it was mentioned before, the initial boundary problem is given by the set of equations $\{(2.1)-(2.4)\}$, where the first equation of the system (2.1) is known the continuity equation in an axisymmetric flow, equation (2.3) define the vorticity $\omega$, equation (2.4) is the vorticity transport equation and equation (2.2) defines the initial-boundary data. To solve this system we will repeat the initial-boundary data in the form

$$
\left.\vec{V}\right|_{t=0}=0 \quad,\left.\quad V_{r}\right|_{z=0}=0 \quad,\left.\quad V_{z}\right|_{z=0}=f(r)=\left\{\begin{array}{lll}
1 & \text { if } & r<a \\
0 & \text { if } & r>0
\end{array}\right.
$$

By using the set of equations (6.3) of initial-boundary data in the computation of calculations for the equations which give the components of the velocity field for old blobs and the components of potential velocity field, we obtained the following results

From figure (1) we observed that the structure of induced velocity field for one vortex blob at arbitrary location is strong around the center of the blob and is weak when it is far from blob. Also, from figure (2) we observed that the same phenomena for the structure of induced velocity field for multi-vortices at different locations which it is strong near the centuries of these blobs and is weak when it far from the blobs. From figure (3) we have the behavior of axial velocity component for potential velocity field at arbitrary points of z-axis. Also, from figure (4) we noticed that the distribution of radial velocity component for potential velocity field like Gaussian distributions. 

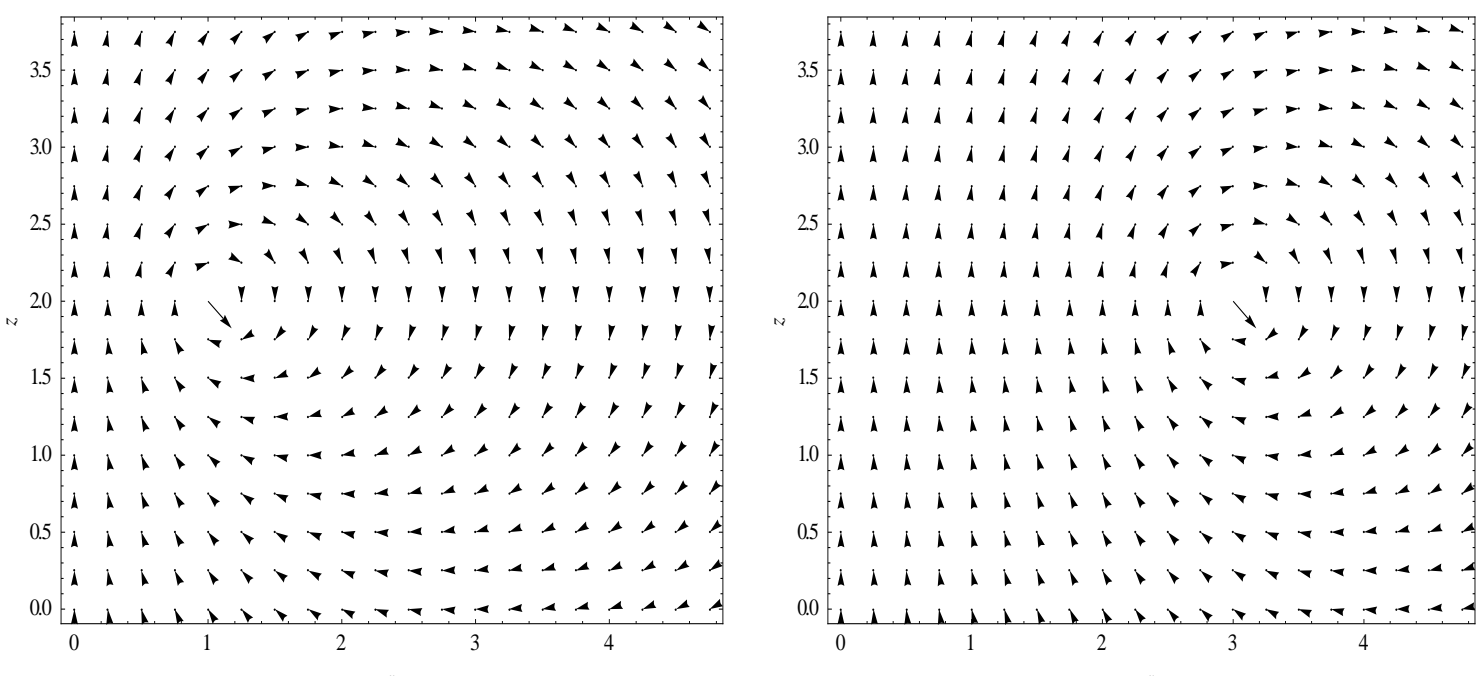

Fig 1:

Induced

velocity field for one vortex blob at different locations

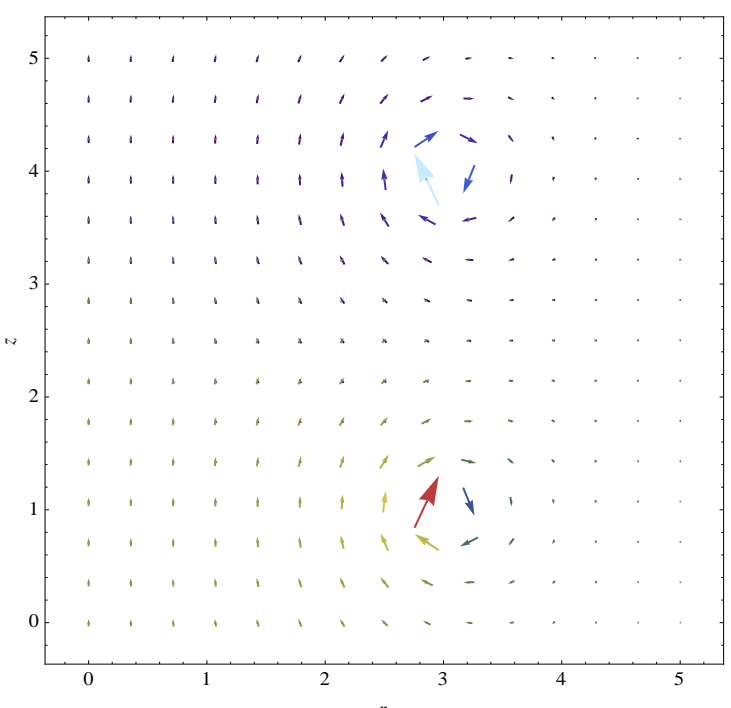

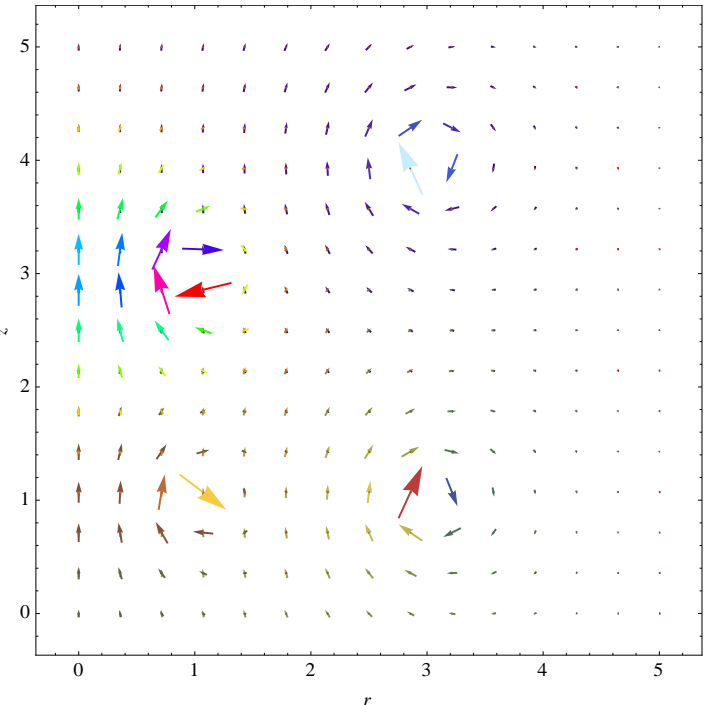

locations
Fig 2:

Induced velocity field for multivortices at different 

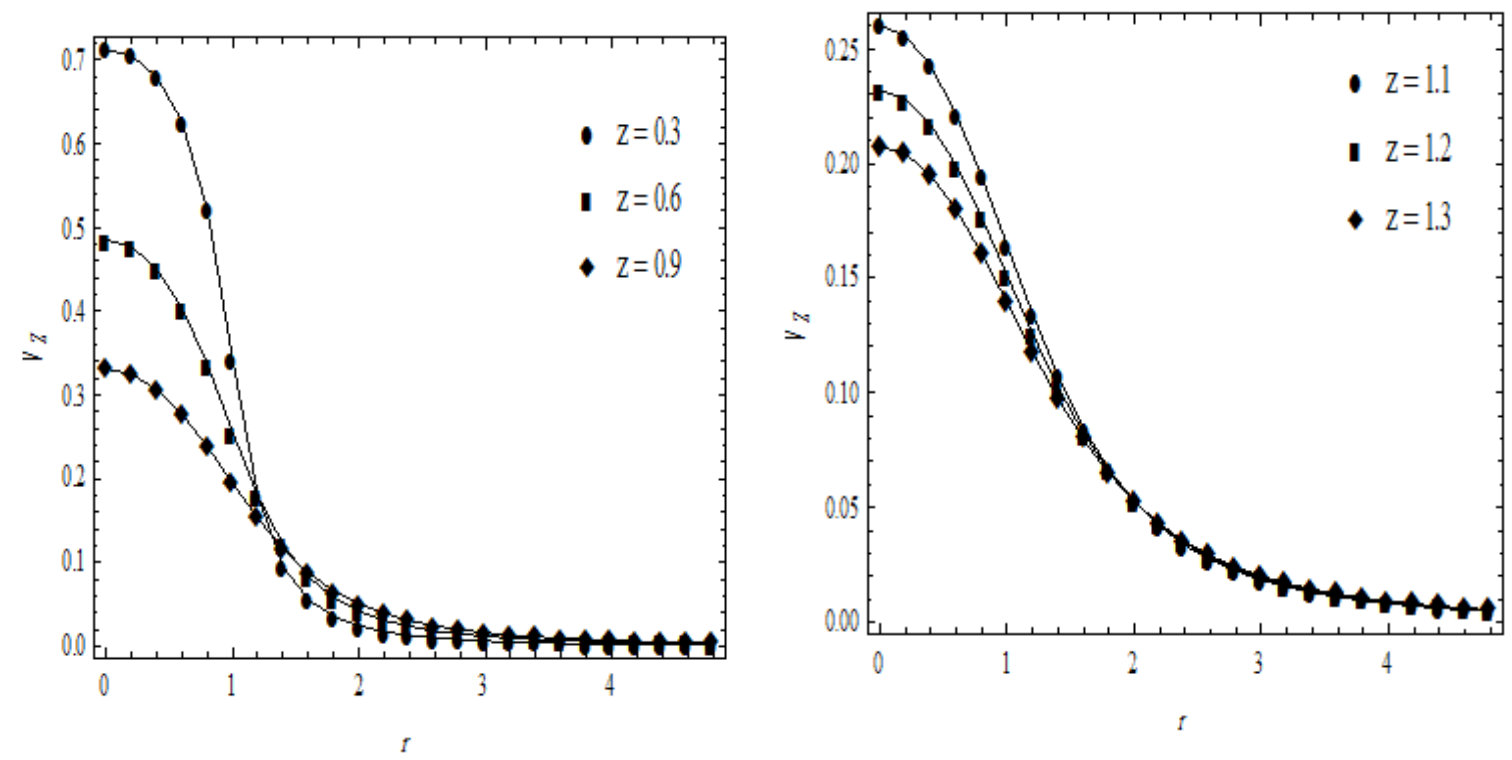

Fig 3: Axial component of potential velocity field at different points
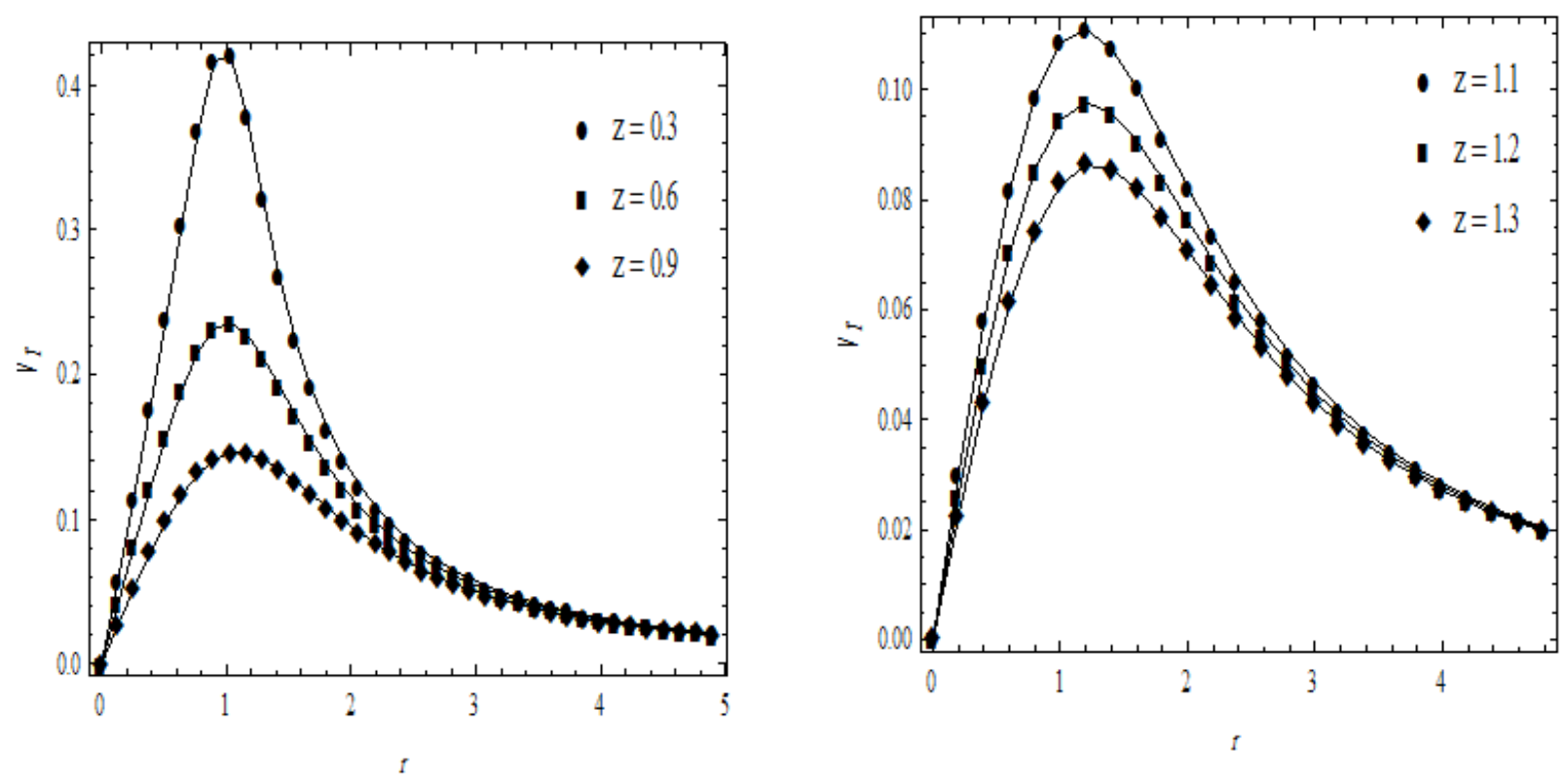

Fig 4: Radial component of potential velocity field at different points

\section{REFERENCES}

[1] Bateman H.: Tables of Integral Transforms. Vol II, McGraw-Hill, Inc., (1954).

[2] Brelot M.: Elements de la theorie classique du potentiel. (russion trans. by E.D.Solomcev, Mir Publish., Moscow), (1964).

[3] Chorin A. J.: Numerical Study of Slightly Viscous Flow. Journal of Fluid Mechanics, 57 (1973), 785-796.

[4] Curtis F. G. and Patrick O. W.: Applied Numerical Analysis. 4th ed., Addison-Wesley Publishing Co., Inc., (1989).

[5] Gardiner C. W.: Handbook of Stochastic Methods for Physics, Chemistry and Natural Science. Springer - Verlag, Berlin Heidelberg New York, 3 rd edition, (1990).

[6] Hedar M. and Styczek A.: Random vortex method approach to axisymmetric jet in a large tank, Journal of Theoretical and Applied Mechanics, 4, 37, (1999), 863-872. 
[7] Modrzewska B. and Styczek A.: Modeling of a Plane Jet via the Vortex Blobs Method. The Archivae of Mechanical Engineering, XXXVIII (1991), 75-86.

[8] Peter A. S.: Introduction to Numerical Methods. Macmillan Publishing Co., Inc., (1970).

[9] Saffman P. G.: Vortex Dynamics. Cambridge University Press, (1992).

[10] Styczek A.: The Vortex Blobs Method of Simulating the Viscous Liquid Motion. The Archivae of Mechanical Engineering, XXXIV (1987), 225-241.

[11] Styczek A., Szumbarski J., and Wald P.: The Stochastic Approach to Viscous Liquid Motion. Proc. of Japanese-Polish Joint Seminar on Advanced Computer Simulation, Tokyo, Japan, Nov. 8 - 9, (1993), 94-102.

[12] Styczek A. and Wald P.: Fast and efficient Vortex-Blobs simulation of the flow past the circular cylinder. The Archive of Mechanical Engineering, XLII (1995), 281-298.

[13] Styczek A. and Szumbarski J.: On the Magnetization-based Lagrangian Methods for 2D and 3D viscous flows. Part I: Theoretical Background, J. Theor. Appl. Mech., 40, 2, (2002), 339-356.

[14] Szumbarski J. and Styczek A.: The Stochastic Vortex Method for Viscous Incompressible Flow in a Spatially Periodic Domain. Arch. Mech., 49, 1, (1997), 209-232. 